\title{
Spontaneous four-wave mixing in optical nanofibers at low temperatures
}

\author{
Anatoly Shukhin ${ }^{1, *}$, and Alexey Kalachev ${ }^{1,2}$ \\ ${ }^{1}$ Kazan E.K. Zavoisky Physical-Technical Institute of the RAS, Kazan, 420029 Russia \\ ${ }^{2}$ Kazan Federal University, Kazan, 420008 Russia
}

\begin{abstract}
In this work, spontaneous four-wave mixing in silica optical nanofibers is studied theoretically. The spectrum of generated biphoton field and its dependence on the nanofiber temperature are analysed.
\end{abstract}

\section{Introduction}

Currently, much attention is paid to developing sources of single photons and entangled photon pairs that are considered as basic ingredients for long-distance quantum communication, optical quantum computing, etc. [1]. One of the most promising approaches to the problem is the use of spontaneous parametric down-conversion and spontaneous fourwave mixing (SFWM) [2]. In particular, SFWM can be observed in widespread third-order nonlinear materials such as silica fibers. In order to increase the effective nonlinear efficiency, we can take advantage of optical micro/nano-fibers (MNF) [3]. However, it is well known that uncorrelated noise from spontaneous Raman scattering of the pump field can significantly decrease coincidence-to-accidental ratio [4]. To solve this problem, the nonlinear material can be cooled to liquid nitrogen [5] or even liquid helium [6] temperature. In the present work, we analyse spectral properties of SFWM in an optical MNF at such low temperatures.

\section{Optical nanofibers}

Fibers with a subwavelength waist diameter are fabricated from standard optical singlemode fibers using heating and stretching method [7]. By adjusting relative movement of fiber and flame during the preparation procedure, almost any adiabatic MNF profile can be made [8].

MNFs have a couple of features which make them pretty good for using as a nonlinear medium for the photon pair generation. First, perfect mode matching between tapered MNF and standard optical fiber can be achieved, which is important when the former is integrated in the telecommunication network. Second, due to the small nanofiber waist diameter, there is a large overlap between all interacting modes, so that efficiency of the SFWM process is high. This fact allows us to decrease the pump power and the length of the nonlinear medium, thereby making photon pair sources compact.

\footnotetext{
*Corresponding author: anatoly.shukhin@mail.ru
} 
SFWM process is a nonlinear effect whereby two photons from the pump field are annihilated and simultaneously two photons in the signal and idler fields, which together called biphoton field, are created. As an example of MNF, we consider a tapered optical fiber with the waist diameter of $950 \mathrm{~nm}$ and waist length of $10 \mathrm{~cm}$. Such transverse waist size is chosen to satisfy the phase matching condition leading to strongly nondegenerate SFWM, when the wavelength of the idler photons belongs to the transparency window of the silica fiber, while that of the signal photons belongs to the visible spectral range where detectors efficiency is high. Standard optical fiber, which MNF is assumed to be made of, has $\mathrm{SiO}_{2}$ core of $10 \mu \mathrm{m}$ diameter and $\mathrm{Ge}$ doped $\mathrm{SiO}_{2}$ cladding of $125 \mu \mathrm{m}$ diameter.

\section{Joint spectral amplitude of the biphoton field}

One of the most important characteristics of the biphoton field is the joint spectral amplitude (JSA) representing the probability amplitude of the photon pair creation as a function of the signal field and idler field wavelengths. In the present work, we study JSA as a function of temperature for MNF and analyse spectral properties of the biphoton field. Fig. 1 presents the results of theoretical simulation. It can be seen that the wavelength of the idler (signal) field decreases (increases) with the temperature, while spectral correlations between the emitted photons do not practically changed.
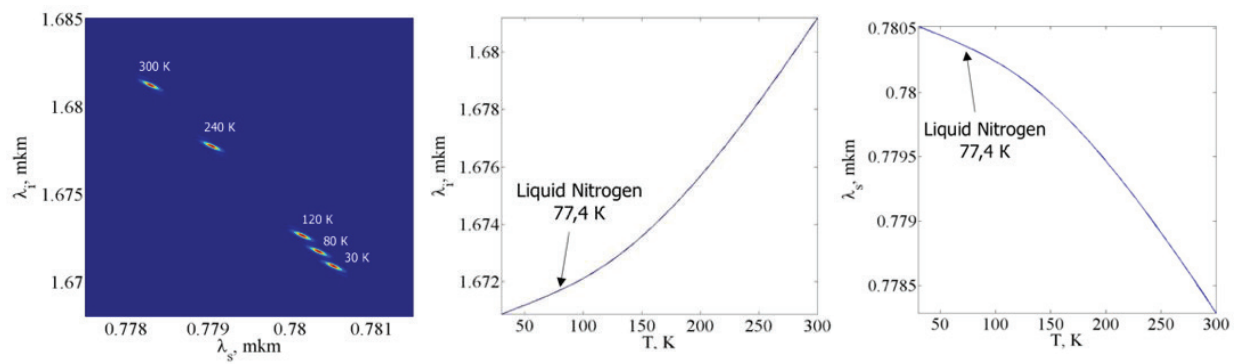

Fig. 1. Joint spectral amplitude of the biphoton field at different temperatures of MNF (left plot) and central wavelength of signal and idler photons as functions of the temperature. Pump wavelength is equal to $1064 \mathrm{~nm}$, while the pump pulse duration is of order of $10 \mathrm{fs}$.

This work is supported by the Program "Topical Problems of Low-Temperature Physics" of the Presidium of the Russian Academy of Sciences.

\section{References}

[1] J. L. O’Brien, A. Furusawa, J. Vučković, Nature Photon. 3, 687 (2009)

[2] M.D. Eisaman et al., Rev. Sci. Instrum. 82, 071101 (2011)

[3] L. Cui et al., Opt. Lett. 38, 5063 (2013)

[4] Q. Lin, F. Yaman, G.P. Agrawal, Phys. Rev. A 75, 023803 (2007)

[5] H. Takesue, K. Inoue, Opt. Express 13, 7832 (2005)

[6] S.D. Dyer, M.J. Stevens, B. Baek, S.W. Nam, Opt. Express 16, 9966 (2008)

[7] L. Tong et al., Opt. Commun. 285, 4641 (2012)

[8] J. M. Ward et al., Rev. Sci. Instrum. 85, 111501 (2014) 